\title{
Homing and Restorative Effects of Bone Marrow-Derived Mesenchymal Stem Cells on Cisplatin Injured Ovaries in Rats
}

\author{
Jiabin Liu ${ }^{1,2,3}$, Haiying Zhang', Yun Zhang ${ }^{2}$, Nan $\mathrm{Li}^{2}$, Yuku Wen ${ }^{2}$, Fanglei Cao ${ }^{2}$, Hao Ai ${ }^{1,2,3, *}$, and Xiaoou Xue ${ }^{4, *}$
}

Premature ovarian failure (POF) is a long-term adverse effect of chemotherapy treatment. However, current available treatment regimens are not optimal. Emerging evidence suggests that bone marrow-derived mesenchymal stem cells (BMSCs) could restore the structure and function of injured tissues, but the homing and restorative effects of BMSCs on chemotherapy injured ovaries are still not clear. In this study, we found that granulosa cell (GC) apoptosis induced by cisplatin was reduced when BMSCs were migrated to granulosa cells (GCs) in vitro. Chemotherapy-induced POF was induced by intraperitoneal injection of cisplatin in rats. BMSCs labeled with enhanced green fluorescent protein (EGFP) were injected into the rats via the tail vein to investigate the homing and distribution of BMSCs in vivo. The number of BMSCs in the ovarian hilum and medulla was greater than in the cortex, but no BMSCs were found in the follicles and corpus lutea. In addition, the BMSCs treatment group's antral follicle count and estradiol levels increased after 30 days, compared with the POF group. Hence, our study demonstrates that intravenously delivered BMSCs can home to the ovaries, and restore its structure and function in POF model rats.

\section{INTRODUCTION}

Chemotherapy regimens cause reproduction damage in young females (Mörse et al., 2013). Premature ovarian failure (POF) is a long-term adverse effect of chemotherapy treatment, which leads to increased risk of infertility and degenerative health

\footnotetext{
${ }^{1}$ Liaoning Medical University, Jinzhou, Liaoning 121001, China, ${ }^{2}$ Department of Gynecology and Obstetrics, Third Affiliated Hospital of Liaoning Medical University, Jinzhou, Liaoning 121001, China, ${ }^{3}$ Key laboratory of Follicular Development and Reproductive Health of Liaoning Province, Liaoning Medical University, Jinzhou, Liaoning 121001, China, ${ }^{4}$ Dongzhimen Hospital Affiliated to Beijing University of China Medicine, Beijing 100029, China

*Correspondence: aihao131@sina.com (HA); xuexiaoou@sina.com (XX)
}

Received 2 June, 2014; revised 22 September, 2014; accepted 23 September, 2014; published online 10 November, 2014

Keywords: bone marrow-derived mesenchymal stem cells, cisplatin homing, intravenous injection, premature ovarian failure conditions, such as cardiovascular disease, osteoporosis, and cognitive impairment (De Vos et al., 2004; Lana et al., 2010; Maclaran and Panay, 2011; Tannock et al., 2004). Therefore, the preservation of fertility and gonadal function has become an important issue for cancer survivors of reproductive age. It has been widely accepted that hormone replacement therapy (HRT), offered to women with POF, could alleviate estrogen deficiency symptoms and minimize the risk of complication (Maclaran and Panay, 2011). However, HRT has been shown to increase the risk of breast cancer (Antoine et al., 2013). Other options, such as ovarian tissue, oocyte and embryo cryopreservation have been applied (Donnez and Dolmans, 2013). But, then again, these available treatment regimens are still not optimal. Therefore, it would be desirable to explore improved therapeutic strategies for treating POF.

Recently, stem cell therapy has been supported as a potential and alternative therapeutic modality, offering the possibility of repairing and restoring the normal function of injured tissues (Agung et al., 2006; Bi et al., 2007; Lee et al., 2005; Muscari et al., 2013). The bone marrow stem cell is the best-studied transplanted stem cell, which contains multiple cell types, such as mesenchymal stem cells (MSCs) and hematopoietic stem cells. MSCs are characterized as adherent cells that has the capability to differentiate into fibroblasts, adipocytes, chondrocytes and osteocytes in vitro (Ohishi and Schipani, 2010). The migration and high proliferation abilities of MSCs ensure that the cells reach the injured tissues to produce protective or restorative effects. Bone marrow-derived mesenchymal stem cells (BMSCs) are promising for regenerative medicine, such as for repairing cardiac, nephritic, osseous and cartilaginous injuries (Agung et al., 2006; Bi et al., 2007; Lee et al., 2005; Muscari et al., 2013).

Previous studies have revealed that bone marrow stem cells can restore ovarian function and generate immature oocytes in female mice (Ghadami et al., 2012; Lee et al., 2007; Selesniemi et al., 2009). It has also been demonstrated that BMSCs can contribute in recovering ovarian structure and function, which were injured by cyclophosphamide (Abd-Allah et al., 2013; Fu et al., 2008; Kilic et al., 2013). In recent years, several other mesenchymal phenotypes, such as adipose-derived MSCs, umbilical cord MSCs, and amniotic fluid MSCs have been referred as a therapeutic potential for chemotherapy induced ovarian damage (Lai et al., 2013; Liu et al., 2012; Takehara et al., 2013; Wang et al., 2013). 
However, selecting an effective method for stem cell delivery is a critical point for achieving a successful cell based therapy. Previous studies have demonstrated that directly injecting BMSCs into the chemotherapy injured ovaries can improve ovarian function and structure (Fu et al., 2008; Kilic et al., 2013). In contrast to cellular orthotopic injection, intravenous injection of BMSCs is a simple, quick and less invasive strategy. More importantly, intravenous injection is specifically more beneficial for chemotherapeutic toxicities that affect various organs. However, there are only a few studies about the homing of BMSCs into the ovaries and the therapeutic potential of intravenous injection for ovaries injured by cisplatin. A current study suggested that intravenous injection of MSCs containing $Y$ chromosomes could migrate into the ovaries and improve ovarian function (Abd-Allah et al., 2013), however, the specific distribution mechanisms of BMSCs in the ovaries were not clear. Therefore, the purpose of the present study is to evaluate the homing and distribution of BMSCs in ovaries injured by cisplatin after intravenous injection, and to evaluate its restorative effects on ovarian structure and function.

\section{MATERIALS AND METHODS}

\section{Animal}

Immature (3-week old) and young (10-week old) female Sprague-Dawley rats were used in the experiments. All animal experiments were approved by the Institutional Animal Committee of Liaoning Medical University.

Isolation, phenotype characterization and labeling of BMSCs BMSCs were obtained from the 10-week-old rats $(n=10)$. Bone marrow was flushed out from tibias using DMEM/F12 (1:1) medium (Hyclone, USA) and centrifuged at 1,000 rpm for $5 \mathrm{~min}$. BMSCs were cultured with a medium, consisting of DMEN/F12 (1:1) medium and $10 \%$ fetal bovine serum (Hyclone), and humidified in a cell culture incubator containing $5 \% \mathrm{CO}_{2}$ at $37^{\circ} \mathrm{C}$. BMSCs were detached with $0.25 \%$ trypsin-EDTA (Hyclone), which was replanted in other flasks with $1: 2$ ratios at $80 \%$ confluence. Third passage BMSCs were used in all experiments.

The surface marker expression of BMSCs was assessed by flow cytometry (Beckman Coulter). BMSCs were suspended (1 $\times 10^{6} \mathrm{cells} / \mathrm{ml}$ ) and stained with FITC- or R-phycoerythrin (PE)conjugated monoclonal antibodies, such as CD29 (Biolegend), CD34 (Santa Cruz), CD45 (eBioscience), CD90 (Biolegend).

The recombinant adenovirus vector with enhanced green fluorescent protein (EGFP) gene (Ad-EGFP) was purchased from SinoGenoMax (China). BMSCs were seeded in 96-well plates, cultured for $24 \mathrm{~h}$, and exposed to Ad-EGFP with a multiplicity of infection (MOI) of 50, 100, 150, 200 and 400 for $12 \mathrm{~h}$. After $48 \mathrm{~h}$, BMSCs were fixed with $4 \%$ paraformaldehyde; and the viability and transfection efficiency of the cells were evaluated by an inverted fluorescence microscope (Olympus IX71, Japan).

Isolation, culture and identification of ovarian granulosa cells

Immature female rats were intraperitoneally injected with pregnant mare serum gonadotropin (Sigma, USA) to stimulate follicle growth. After $48 \mathrm{~h}$, bilateral ovaries were removed under aseptic conditions. Then, the adipose and connective tissues of the ovaries were removed and washed twice with PBS solution. GCs were isolated under an anatomical microscope and single cell suspensions were obtained. The cells were washed three times and centrifuged at 1,000 rpm for $5 \mathrm{~min}$. The GCs were cultured with a medium, consisting of a DMEN/F12 (1:1) medi- um and $10 \%$ fetal bovine serum with $5 \% \mathrm{CO}_{2}$ at $37^{\circ} \mathrm{C}$. First passage GCs were used in all experiments.

The follicle stimulating hormone receptor (FSHR) of the GCs was assessed by immunocytochemistry. GCs were seeded in 24-well plates (CORNING, USA), cultured for $48 \mathrm{~h}$, fixed with $4 \%$ paraformaldehyde for $30 \mathrm{~min}$, and washed twice with PBS solution. Then, the GCs were incubated with $3 \% \mathrm{H}_{2} \mathrm{O}_{2}$ for 10 min at room temperature to eliminate endogenous catalase activities. Subsequently, the GCs were incubated in a humidified chamber for one hour at $37^{\circ} \mathrm{C}$ with FSHR primary monoclonal antibodies (1:100, Santa Cruz). Negative control was conducted with PBS alone. After incubating with Polymer Helper (ZSBIO, China) for $20 \mathrm{~min}$, polyperoxidase-anti-rabbit lgG (ZSBIO, China) were added for $20 \mathrm{~min}$ at $37^{\circ} \mathrm{C}$. Then, color developed with the 3,3'-Diaminobenzidine (DAB) chromogen. After washing with tap water, the samples were determined by an Olympus IX71 microscope.

Effects of cisplatin and BMSCs on apoptotic GCs in vitro The toxicity of cisplatin (Qilu Pharmaceutical Ltd. China) to GCs was assessed by Cell Counting Kit-8 (CCK-8, Dojindo Company, Japan). The GCs were seeded at a density of $10^{4}$ cells in 96-well plates, cultured for $72 \mathrm{~h}$, and exposed to various cisplatin concentrations $(0,1.0,5.0$ and $7.5 \mathrm{mg} / \mathrm{L})$. After $48 \mathrm{~h}, 100$ microliters of medium, consisting of DMEN/F12 (1:1) medium and 10\% CCK-8 without fetal bovine serum, was added to each well and incubated for $4 \mathrm{~h}$. Then, OD value was assessed by a microplate reader (Awareness Technology). After the supernatant was removed, the GCs were fixed with $4 \%$ paraformaldehyde for $30 \mathrm{~min}$ and stained with crystal violet (Sigma, USA).

The GCs were divided into three groups: normal contro group, cisplatin $(5 \mathrm{mg} / \mathrm{L})$ group, and cisplatin $(5 \mathrm{mg} / \mathrm{L}) \mathrm{co}-$ cultured with BMSCs group. The GCs $\left(2.5 \times 10^{5}\right.$ cells/well $)$ were seeded in 6-well plates and cultured for $48 \mathrm{~h}$. In the cisplatin and cisplatin co-cultured with BMSCs groups, GCs were cultured with $2 \mathrm{ml}$ medium, which included $5 \mathrm{mg} / \mathrm{L}$ of cisplatin. In the cisplatin co-cultured with BMSCs group, BMSCs were seeded on the upper side of the Transwell chambers $(0.4$ $\mu \mathrm{m}$, CORNING) at a density of $2.5 \times 10^{5}$ cells/well. After $48 \mathrm{~h}$, the GCs were collected and assessed by flow cytometry (Beckman Coulter) with an Annexin V/PI apoptosis detection kit (eBioscience, USA).

\section{Migration of BMSCs in vitro}

The migration of BMSCs was evaluated by Transwell Permeable Supports (CORNING) with $8.0 \mu \mathrm{m}$ pore filters (Rüster et al., 2005). The GCs were divided into two groups: normal control group and cisplatin $(5 \mathrm{mg} / \mathrm{L})$ group. The GCs $\left(1 \times 10^{5} /\right.$ well $)$ were seeded in 24-well plates and cultured for 24 hours. In the cisplatin group, the GCs were incubated with a $500 \mu \mathrm{l}$ medium and cisplatin ( $5 \mathrm{mg} / \mathrm{L})$. After $48 \mathrm{~h}, 200 \mu \mathrm{l}$ of medium, containing BMSCs $\left(5 \times 10^{4}\right.$ cells $)$, was added on the upper side of the Transwell chambers $(8.0 \mu \mathrm{m}$, CORNING). The plates were incubated for 6 and $24 \mathrm{~h}$. BMSCs were removed from the upper side of the filters; BMSCs at the lower side of filters were fixed with $4 \%$ paraformaldehyde for $20 \mathrm{~min}$, and stained with crystal violet. The number of migrated BMSCs was determined by counting five random fields per well using an Olympus IX71 microscope.

POF model and intravenous injection of BMSCs The animals were divided into three groups (10 rats/group): normal control group (group 1), cisplatin-induced POF group (group 2) and BMSCs treatment group (group 3). To establish 
the POF model, the rats were intraperitoneally injected with a daily dose of cisplatin at $2 \mathrm{mg} / \mathrm{kg}$ of body weight for six days. BMSCs $\left(4 \times 10^{6}\right.$ cells/rat) labeled with EGFP in $0.6 \mathrm{ml}$ PBS were injected via the tail vein on the seventh day. At day 15 and 30 , five rats from each group were narcotized; the blood and organs were collected. The serum was isolated, and stored at $80^{\circ} \mathrm{C}$ for hormone test. The ovaries and other organs were dissected immediately, and were fixed in $4 \%$ paraformaldehyde for follicle counting, GFP-labeled BMSCs tracking and TUNEL.

\section{Estradiol level}

Blood was collected from postcava and centrifuged at 3,000 rpm for $10 \mathrm{~min}$ to separate the serum. The level of estradiol $\left(E_{2}\right)$ was measured with an ARCHITECT Estradiol Reagent Kit (Abbott, USA).

\section{Ovarian morphologic analysis}

The left ovary of each rat was fixed with $4 \%$ paraformaldehyde for $24 \mathrm{~h}$, embedded in paraffin, cut into sections $(5 \mu \mathrm{m})$, and stained with hematoxylin and eosin (HE) -- to evaluate follicle growth. Preantral follicles contained less than five layers of GCs and lacked antral vesicles. Antral follicles contained more than five layers of GCs and antral vesicles. The antral follicles and corpus were counted in the biggest section; which was across the ovarian hilum. The sections were examined and photographed with the Olympus BX-51 light microscope (Olympus, Japan).

\section{Tracking BMSCs by immunohistochemical staining} The organs were fixed in $4 \%$ paraformaldehyde for $24 \mathrm{~h}$, dehydrated and embedded in paraffin. Sections $(5 \mu \mathrm{m})$ were deparaffinized, rehydrated and high pressured for $2 \mathrm{~min}$ in a citrate buffer ( $\mathrm{pH} \mathrm{6.0)}$ to retrieve antigenicity. Then, the samples were incubated with $3 \%$ $\mathrm{H}_{2} \mathrm{O}_{2}$ for 10 min at room temperature. The samples were incubated in a humidified chamber for one hour at $37^{\circ} \mathrm{C}$ with EGFP primary monoclonal antibodies (1:100, Santa Cruz). Negative control was conducted with PBS alone. After incubating with Polymer Helper (ZSBIO, China) for $20 \mathrm{~min}$, polyperoxidase-anti-rabbit IgG (ZSBIO, China) were added for $20 \mathrm{~min}$ at $37^{\circ} \mathrm{C}$. Then, color developed with the 3,3'-Diaminobenzidine (DAB) chromogen and was counterstained with hematoxylin for $20 \mathrm{~s}$. After washing with tap water, the samples were dehydrated and photographed with the Olympus BX51 microscope.

\section{TUNEL assay}

Ovarian granulosa cell apoptosis was measured by TUNEL apoptosis assay kit (Promega, USA), according to manufacturer's instructions. The ovarian sections, across ovarian hilum and nuclei of apoptotic cells, were stained dark brown. The sections were examined and photographed with the Olympus BX-51 light microscope.

\section{Statistical analysis}

To calculate the statistical differences between the groups, the SPSS 19.0 statistical package (Chicago, USA) was used. One-way ANOVA was used to determine the significant differences among the groups. All values were presented as mean \pm standard deviation. In general, $P$ values $<0.05$ were considered significant.

\section{RESULTS}

Characterization and labeling of BMSCs

After isolating from the bone marrow and $48 \mathrm{~h}$ of adherent growth, the BMSCs morphologically resembled fibroblasts. The

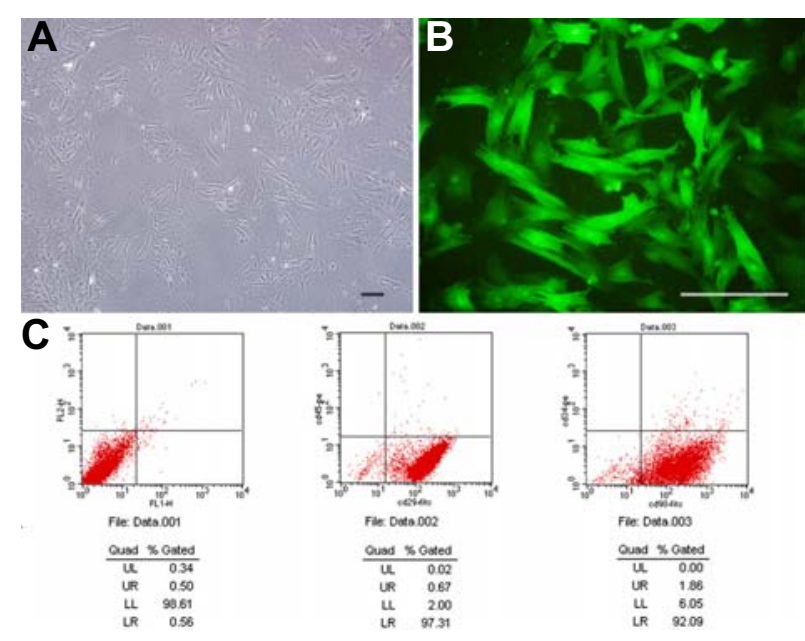

Fig. 1. Morphology and phenotypes of BMSCs: (A) Third passage BMSCs showed fibroblastic morphology; (B) Fluorescent microscopic view of BMSCs transfected with Ad-EGFP; (C) Flow cytome try revealed that $\mathrm{BMSC}$ s were $\mathrm{CD} 29^{+}, \mathrm{CD}^{+} 0^{+}$and $\mathrm{CD} 34^{-}, \mathrm{CD} 45^{-}$. Scale bars, $20 \mu \mathrm{m}$.

morphology of third passage BMSCs was consensus (Fig. 1A). Surface antigens of the BMSCs were characterized by flow cytometry. Most of the BMSCs were CD29 ${ }^{+}, \mathrm{CD}^{+}{ }^{+}$and $\mathrm{CD} 34^{-}$, $\mathrm{CD}^{-} 5^{-}$(Fig. 1C). To trace the fate of the BMSCs in vivo, the BMSCs were labeled with Ad-EGFP. After transfection, the green fluorescence protein started to express after $48 \mathrm{~h}$; and highly expressed after $72 \mathrm{~h}$. When the BMSCs were transfected with an $\mathrm{MOI}$ of $200,99 \%$ of the BMSCs expressed green fluorescence proteins (Fig. 1B).

\section{Characterization of GCs}

After isolating the GCs from the ovarian follicles, the cells showed adherent growth and morphologically resembled round or oval cells (Fig. 2A). The morphology of the first passage GCs was consensus, containing only a few polygonal cells. The GCs were characterized by immunocytochemistry. The round or oval cells expressed as intracellular follicle stimulating hormone receptor (FSHR), while the polygonal cells were negative (Fig. 2C).

GCs injured by cisplatin and protected by BMSCs

To determine the effects of the viability and inhibition of GCs induced by cisplatin, the viability of the GCs were measured by CCK-8 assay. Significant cytotoxic effects and cell growth inhibition on the GCs were shown by cisplatin; the GCs were sparse and irregular (Fig. 2B). Compared with the control group, the viability of GCs with high doses of cisplatin (5.0 and 7.5 $\mathrm{mg} / \mathrm{L}$ ) significantly decreased, compared to normal conditions $(P<0.01)$ (Fig. 2D). When co-cultured with BMSCs, apoptosis was reduced in GCs. As shown in Fig. 3, live GCs increased from $68.51 \%$ to $78.47 \%$ in the cisplatin and BMSCs groups (Figs. 3B and 3C).

\section{BMSCs migrated to GCs injured by cisplatin}

The migration ability of BMSCs was assessed by Transwell assay. After $24 \mathrm{~h}$, an abundant number of BMSCs migrated to GCs that were injured by cisplatin, through polycarbonate filters (Fig. 4B). Only a few BMSCs migrated to the normal GCs (Fig. 4A). Statistical analysis of the number of BMSCs revealed that GCs injured by cisplatin promoted BMSCs to migrate after 6 

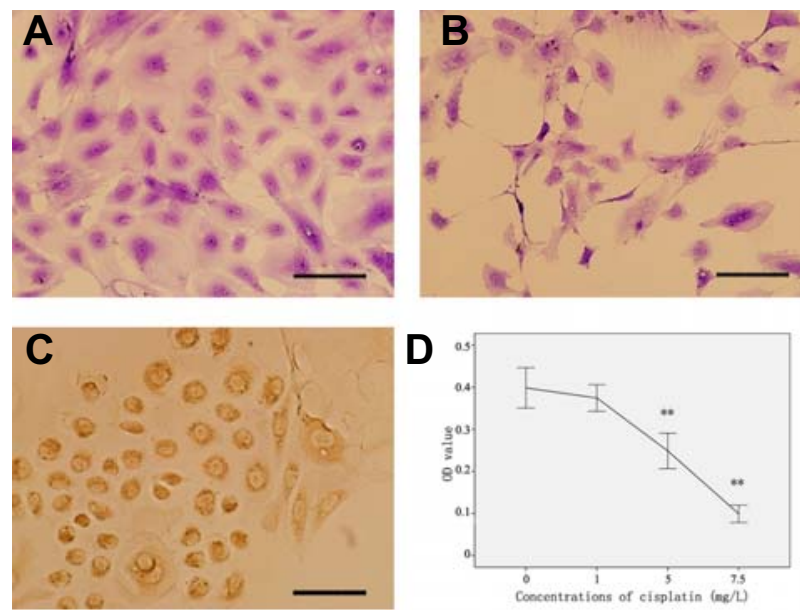

Fig. 2. Morphology and phenotypes of GCs, and the effects of cisplatin on GCs: (A) The morphology of normal first passage GCs; (B) The morphology of GCs injured by cisplatin; (C) FSHR expression in GCs; (D) CCK-8 assays indicated that cisplatin significantly injured GCs, compared to normal, ${ }^{\star \star} P<0.01$. Scale bars, $20 \mu \mathrm{m}$.

and $24 \mathrm{~h}$ (Fig. 4C).

\section{Estradiol levels}

30 days after injecting BMSCs, the $E_{2}$ serum in the BMSCs group increased compared with the POF group $(P<0.01)$. However, the $E_{2}$ serum in the BMSCs group diminished compared with the normal group $(P<0.05)$. There was no significant difference between the BMSCs group and POF group on the $15^{\text {th }}$ day (Fig. 5A).

\section{Histological examination and follicle count}

All stages of follicular development (primary, secondary, and antral follicles) were observed in the rat control group for 30 days (Fig. 6A). Specimens that were taken from the POF group had only a few primordial follicles with degenerated growth and antral follicles (Fig. 6B). All types of follicles, including the pri-
A,

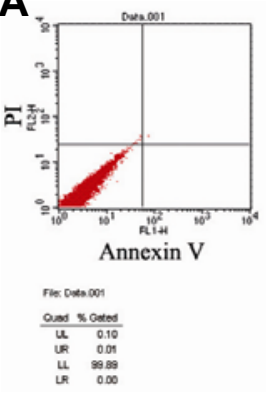

B
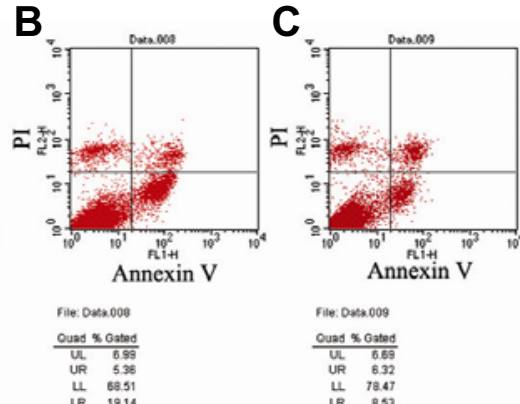

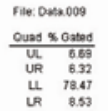

Fig. 3. Co-culture with BMSCs, induced apoptosis in GCs: (A) Normal GCs; (B) Apoptotic rate of GCs in the cisplatin group; (C) Co-cultured with BMSCs.

mary, secondary and antral, and corpus luteum were apparently normal in the BMSCs treatment group (Figs. 6C and 6D). In addition, the antral follicles in the treatment group were healthy (Figs. 6E and 6F).

The statistical analysis of the antral follicular count revealed that the increased dosage of cisplatin resulted to the decreased amount of follicular count. However, in the BMSCs treatment group, a significant increase in healthy antral follicles and corpus lutea count was detected, compared with the cisplatin injection group (Fig. 5B); no significant change was observed after 15 days $(P>0.05)$.

\section{Detecting BMSCs by immunohistochemistry}

Following the transplantation of BMSCs, ovaries displayed EGFP-positive BMSCs on the $15^{\text {th }}$ day. Interestingly, the number of BMSCs in the ovarian hilum and ovarian medulla was greater than in the ovarian cortex (Figs. 7A-7F). We also found BMSCs surrounding the follicles; but there were no BMSCs in the ovarian follicle and corpus lutea. 30 days after injecting BMSCs, we found that BMSCs survived and proliferated in the ovary (Figs. 7G-7I). BMSCs were also found in other organs, such as the heart, kidneys, liver and adipose tissues near the ovaries (Figs. 7J-7L). However, the number of BMSCs in the ovaries and kidneys were greater than in the liver and heart.
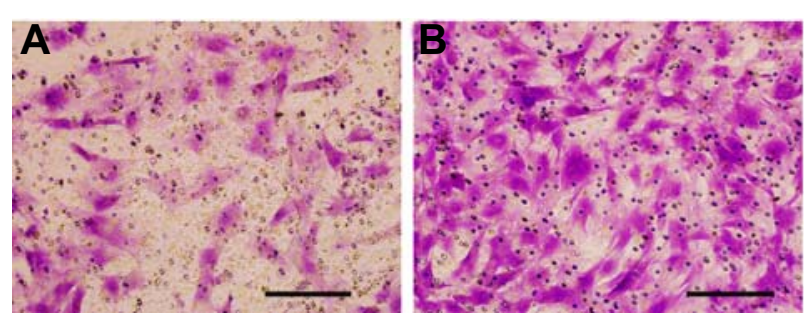

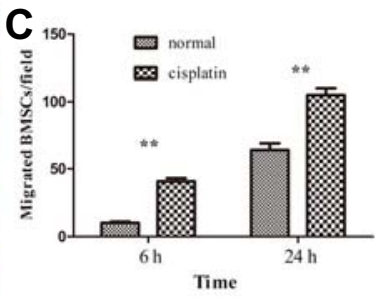

Fig. 4. BMSCs migrated to cisplatin injured GCs: (A) BMSCs migrated to normal GCs in $24 \mathrm{~h}$; (B) BMSCs migrated to cisplatin injured GCs; (C) The number of migrated BMSCs every $200 \times$ magnification field $(n=$ 5), ${ }^{\star \star} P<0.01$. Scale bars, $20 \mu \mathrm{m}$.
A

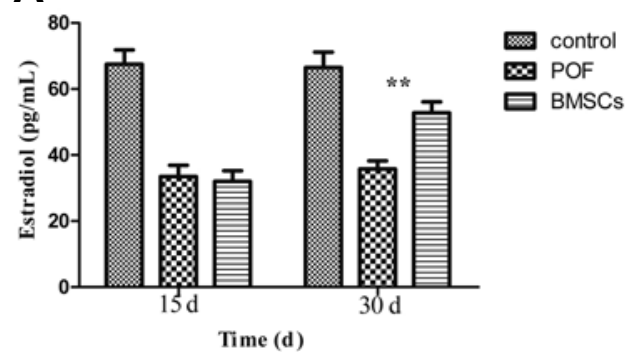

B

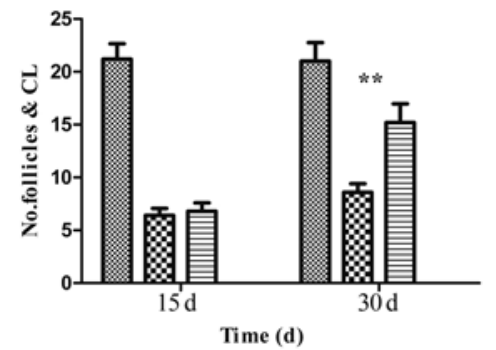

Fig. 5. Serum levels of estradiol $\left(E_{2}\right)$, the number of antral follicles and corpus lutea in the three groups (control, POF, and BMSCs treated; $n=5$ ), 15 and 30 days after administering BMSCs. Data were expressed as means $\pm S D$ in numerous experiments; 15 days (control vs. POF $P<$ 0.01 ; control vs. BMSCs $P<0.01$; POF vs. BMSCs $P>0.05$ ); 30 days (control vs. POF $P<0.01$; control vs. BMSCs $P<$ 0.05; POF vs. BMSCs $\left.{ }^{* *} P<0.01\right)$. 

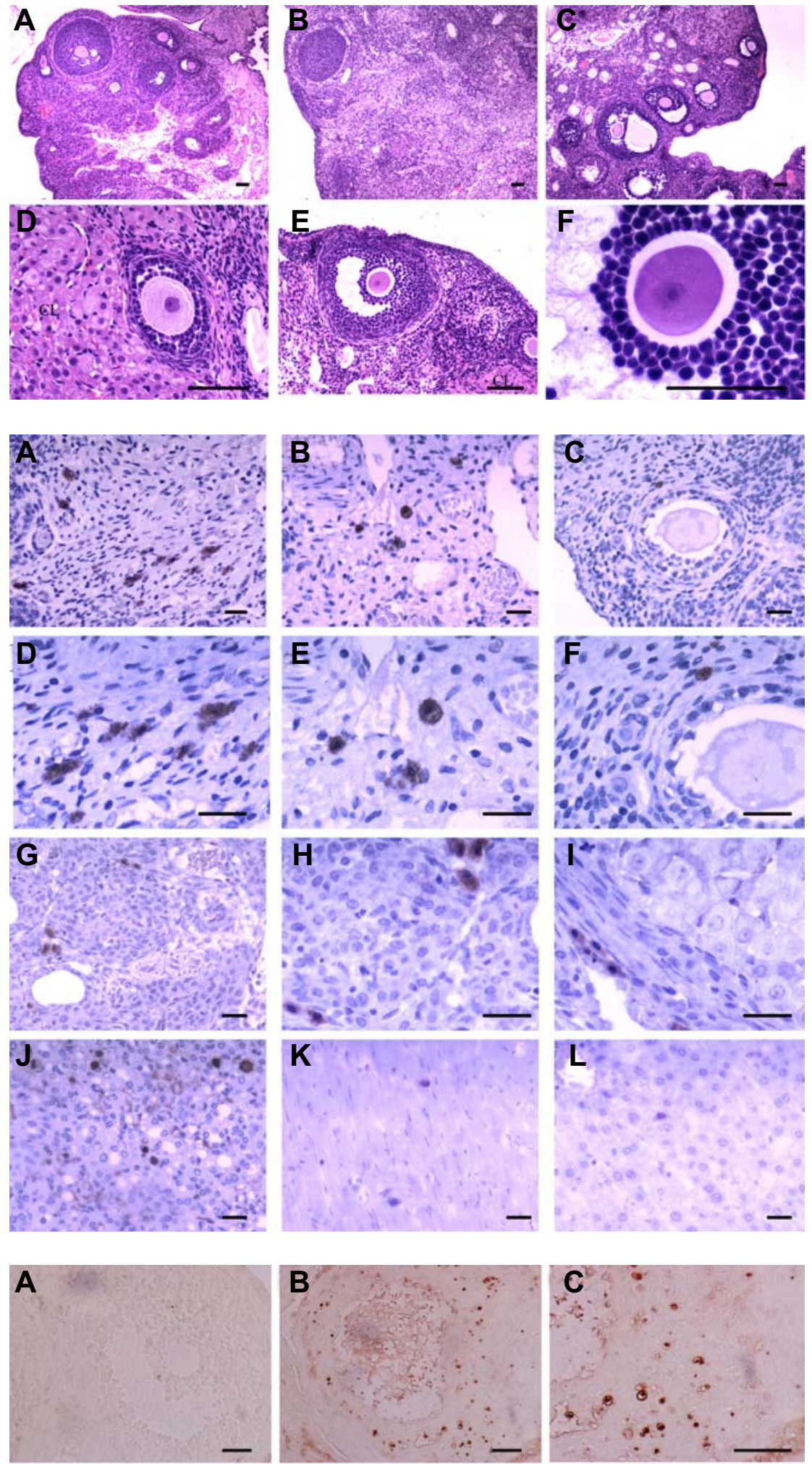

Fig. 6. Histologic analysis of the ovaries after transplanting BMSCs. The ovaries of the control group (A), POF group (B), and BMSCs treated group (C); (D-F) the primary follicle, secondary follicle, antral follicle and corpus luteum $(\mathrm{CL})$ of the BMSCs treated group. Scale bars, 100 $\mu \mathrm{m}$.

Fig. 7. EGFP-positive BMSCs in tissue sections: (A-F) transplanted BMSCs in the ovaries after 15 days; $(A, D)$ BMSCs in the ovarian hilum; $(B, E)$ BMSCs in the ovarian medulla; (C, F) BMSCs in the ovarian cortex, surrounding follicles; (G-I) transplanted BMSCs in the ovaries after 30 days; $(G, H)$ BMSCs surrounding follicles, (I) BMSCs surrounding corpus lutea; (J-L) BMSCs in the kidneys, heart and liver. Scale bars, $50 \mu \mathrm{m}$.

Fig. 8. Apoptosis of GCs in the ovaries: (A) no apoptotic GCs were observed in the healthy antral follicles; $(B, C)$ apoptotic GCs were observed in the atretic follicles. Scale bars, $50 \mu \mathrm{m}$.
More interesting, most of the BMSCs were distributed along the blood vessels.

Granulosa cell apoptosis in antral follicles

The granulosa cell apoptosis was detected by TUNEL. There were no apoptotic GCs in the healthy antral follicles (Fig. 8A). An abundant number of apoptotic GCs were observed in the atretic follicles that did not contain oocytes (Figs. 8B and 8C). Atretic follicles were found in all groups; and the number of healthy antral follicles in the BMSCs group was greater than in 
Homing and Restorative Effects of BMSCs on Damaged Ovaries

Jiabin Liu et al.

the POF group after 30 days (healthy antral follicular count $5.60 \pm$ 1.14 in BMSCs group versus $2.60 \pm 1.52$ in POF group; $P<0.01$ ).

\section{DISCUSSION}

The ability of BMSCs to self-renew and regenerate pushed the trial to evaluate their role in fertility preservation. So far, no ideal marker for BMSCs has been identified. However, it has been identified that BMSCs expressed CD29, CD90, CD105 and negative expressions of hematopoietic cell-surface antigens, such as CD45, CD34, CD14 (Ohishi and Schipani, 2010). In our study, BMSCs were identified to express CD90 and CD29, but not CD45 and CD34. In addition to their fibroblast-shaped morphology and adherence to plastic, it was possible to conclude that the cell population used in our study was an enriched culture of BMSCs. Since homing of BMSCs is inefficient and many BMSCS were trapped in the lung following systemic administration, it is imperative that we trace the fate of the injected cells. We transfected BMSCs with Ad-EGFP and yielded the highest percentage of GFP-expression cells (99\%) without affecting cell viability (Bosch et al., 2006; Yuan et al., 2011). A classic method in labeling cells is with Ad-EGFP; which expresses fluorescent proteins that are helpful in gaining insights on the homing and engraftment of BMSCs.

Cisplatin is one of the most effective chemotherapeutic agents, but injury may occur at higher dosages. It has been reported that cisplatin caused ovarian failure during clinical usage and nonclinical investigations (Ai et al., 2012; lorio et al., 2014; Nozaki et al., 2009). Cisplatin induced granulosa cell apoptosis or necrosis in vitro (Guo et al., 2013). This cytotoxicity depended on the concentration of cisplatin. The cytotoxicity of cisplatin was considered based on several factors, such as oxidative stress, energetic metabolism impairment, and DNA injury (Santos et al., 2007; Siddik, 2003). In this study, after cisplatin was administered through intraperitoneal injections, the rats showed only a few healthy antral follicles, but more atretic follicles. Other than premature ovarian failure, cisplatin also caused other toxicities, such as nephrotoxicity, myelosuppression, and hepatotoxicity (Bi et al., 2007; Das et al., 2008; Miyamoto et al., 2007; Santos et al., 2007; Yuan et al., 2011). In addition, previous studies have shown that BMSCs could restore various injured organs, such as bone, cartilage, heart, kidneys and ovaries. Therefore, we selected intravenous injection as the cell delivery method.

In the present study, BMSCs that migrated to granulosa cells injured by cisplatin in vitro and the amount of cells in the ovaries and kidneys injured by cisplatin were greater, compared with other organs, such as the liver and heart. The reason for the uneven distribution of BMSCs in the body may be related to the varying degrees of damage induced by cisplatin to the organs. For example, cisplatin induced hepatotoxicity was slight, while its nephrotoxicity was more extensive (Miyamoto et al., 2007). In the ovaries, BMSCs were mainly distributed in the ovarian hilum and medulla, a few in the ovarian cortex, and none in the follicle and corpus lutea. We also found that BMSCs was mainly distributed along the blood vessels. The rich blood vessels in the ovarian hilum and medulla might explain the uneven distribution of BMSCs in the ovaries. Many investigations suggested that BMSCs migrated to the injured tissues, which contributed to tissue regeneration. However, the precise cell homing mechanisms still remains to be unknown. MSCs express many receptors and cell adhesion molecules that aids in migration and homing to target tissues, such as CXCL12 and receptor CXCR4, CCL21 and receptor CCR7, and integrin (Sohni and Verfaillie, 2013). Migration and homing requires cells to have the ability to attach and migrate between endothelial cells (ECs) in order to enter the target tissue. It is well established that leukocytes attach to ECs, roll over the ECs, and transmigrate between ECs (Sohni and Verfaillie, 2013); and there are also other factors that promote MSCs to migrate, such as TNF- $\alpha$, HGF and basic fibroblast growth factors (Forte et al., 2006; Schmidt et al., 2006; Zhang et al., 2010).

The present study found that the infusion of BMSCs could restore ovarian function. 30 days after administering BMSCs, ovarian function and structure improved based on results; which showed increased estradiol levels and follicle population The mechanisms that enable BMSCs to induce beneficial effects in the ovaries are still controversial. It has been proposed that BMSCs can differentiate into fibroblasts, adiopocytes, chondrocytes, and osteocytes in vitro. Thus, the differentiation ability of BMSCs is promising for regenerative medicine applications. In addition, bone marrow transplantation into female mice generated a few donor-derived immature follicles (Johnson et al., 2005; Lee et al., 2007). Similarly, it has also been reported that spermatogonia could be derived from the bone marrow of a male mice (Lue et al., 2007; Nayernia et al., 2006). In this study, no EGFP-positive BMSCs were observed in the follicles and corpus lutea. EGFP-positive BMSCs were mainly distributed in the ovarian hilum and medulla, and in the thecal layers of the ovarian cortex. These results showed that BMSCs may not differentiate into granulosa cells (GCs) and oocytes, but may engraft in the ovarian interstitium -- resulting to restorative effects on follicle development. Another study has also revealed that adipose-derived MSCs were not located in the follicles, but in the thecal layers (Takehara et al., 2013). This observation indicates that the ability of BMSCs to repair cisplatin injured ovaries was not caused by their differentiation, but by other mechanisms that might exist.

It has been identified that follicular growth needed the systemic regulation by hormones and intraovarian regulation by cytokines, growth factors, and intracellular proteins (Matsuda et al., 2011). Granulosa cells, in particular, play an important role in follicular development and maintenance (Maruo et al., 1999). In our study, BMSCs reduced the apoptosis rate of cisplatin injured GCs in vitro, and the intravenous injection of BMSCs restored the number of healthy follicles and induced follicular atresia. We also found that the apoptosis of GCs only occurred in the large secondary and antral follicles. This suggests that regulating GCs could protect the follicles. The restorative effects of BMSCs on GCs and follicles may probably be due to their secretion ability, such as growth factors, angiogenic factors, pleiotropic cytokines, chemotactic cytokines, and extracellular matrix proteins (Kupcova Skalnikova, 2013). It has been demonstrated that MSCs secreted a variety of factors, such as epidermal growth factor (EGF), vascular endothelial growth factor (VEGF), insulin like growth factor I (IGF-I), hepatocyte growth factor (HGF), insulin-like growth factor-binding proteins, and transforming growth factor b (Kupcova Skalnikova, 2013). Among those mentioned, VEGF, HGF, and IGF-1 have been demonstrated to restore ovarian function and structure (AbdAllah et al., 2013; Fu et al., 2008). These findings suggest that the secretions of BMSCs may alter tissue microenvironment and promote functional recovery.

Therefore, the migration capabilities of BMSCs to injured tissue sites and its secretion function, allows BMSCs to exert their restorative actions. Moreover, studies have shown that BMSCs not only protects the ovaries from cisplatin-induced injuries, but also protects and restores other organs that were injured by cisplatin, such as kidneys and the bone marrow (Bi et al., 2007; 
Frenette et al., 2013). These results suggest that systemically delivered BMSCs are fit for protecting and restoring multiple organs injured by chemotherapeutic toxicities.

In conclusion, the present study demonstrated that through intravenous injection, BMSCs home to the ovaries and distribute mainly in the ovarian hilum and medulla; a small number of BMSCs engraft in the ovarian cortex; and no BMSCs were observed in the follicles and corpus lutea. In addition, ovarian function and structure was restored, after intravenously injecting BMSCs. Therefore, intravenously injecting BMSCs is an efficient treatment method for chemotherapy-induced POF.

\section{ACKNOWLEDGMENTS}

This study was supported by grants from the National Natural Science Foundation of China (81173443), Scientific Research Fund of Liaoning Provincial Education Department (LJQ2011087), Liaoning BaiQianWan Talents Program (2009921040), Liaoning province Science and Technology Program (2009225010-47; 2013225305), and Aohongboze Graduate Sci-tech Innovation Foundation, The President Fund of Liaoning Medical University (2013008). We declare no potential conflicts of interest.

\section{REFERENCES}

Abd-Allah, S.H., Shalaby, S.M., Pasha, H.F., El-Shal, A.S., Raafat, N., Shabrawy, S.M., Awad, H.A., Amer, M.G., Gharib, M.A., El Gendy, E.A., et al. (2013). Mechanistic action of mesenchymal stem cell injection in the treatment of chemically induced ovarian failure in rabbits. Cytotherapy 15, 64-75.

Agung, M., Ochi, M., Yanada, S., Adachi, N., Izuta, Y., Yamasaki, T., and Toda, K. (2006). Mobilization of bone marrow-derived mesenchymal stem cells into the injured tissues after intraarticular injection and their contribution to tissue regeneration. Knee Surg. Sports Traumatol. Arthrosc. 14, 1307-1314.

Ai, H., Zhang, H., Zhang, Y., and Liu, Y. (2012). Mouse ovarianrelated gene expression profiles change with intraperitoneal injection of cisplatin. African J. Pharm. Pharm. 6, 2119-2122.

Antoine, C., Ameye, L., Paesmans, M., and Rozenberg, S. (2013). Systematic review about breast cancer incidence in relation to hormone replacement therapy use. Climacteric 17, 116-135.

Bi, B., Schmitt, R., Israilova, M., Nishio, H., and Cantley, L.G. (2007). Stromal cells protect against acute tubular injury via an endocrine effect. J. Am. Soc. Nephrol.18, 2486-2496.

Bosch, P., Fouletier-Dilling, C., Olmsted-Davis, E., Davis, A., and Stice, S. (2006). Efficient adenoviral-mediated gene delivery into porcine mesenchymal stem cells. Mol. Reprod. Dev. 73, 13931403.

Das, B., Antoon, R., Tsuchida, R., Lotfi, S., Morozova, O., Farhat, W., Malkin, D., Koren, G., Yeger, H., and Baruchel, S. (2008). Squalene selectively protects mouse bone marrow progenitors against cisplatin and carboplatin-induced cytotoxicity in vivo without protecting tumor growth. Neoplasia 10, 1105-1119.

De Vos, F., Nuver, J., Willemse, P., van der Zee, A., Messerschmidt, J., Burgerhof, J., De Vries, E., and Gietema, J. (2004). Longterm survivors of ovarian malignancies after cisplatin-based chemotherapy: cardiovascular risk factors and signs of vascular damage. Eur. J. Cancer 40, 696-700.

Donnez, J., and Dolmans, M.-M. (2013). Fertility preservation in women. Nat. Rev. Endocrinol. 9, 735-749.

Forte, G., Minieri, M., Cossa, P., Antenucci, D., Sala, M., Gnocchi, V., Fiaccavento, R., Carotenuto, F., De Vito, P., Baldini, P.M., et al. (2006). Hepatocyte growth factor effects on mesenchymal stem cells: proliferation, migration, and differentiation. Stem Cells 24, 23-33.

Frenette, P.S., Pinho, S., Lucas, D., and Scheiermann, C. (2013). Mesenchymal stem cell: keystone of the hematopoietic stem cell niche and a stepping-stone for regenerative medicine. Ann. Rev. Immunol. 31, 285-316.

Fu, X., He, Y., Xie, C., and Liu, W. (2008). Bone marrow mesenchymal stem cell transplantation improves ovarian function and structure in rats with chemotherapy-induced ovarian damage. Cytotherapy 10, 353-363.
Ghadami, M., El-Demerdash, E., Zhang, D., Salama, S.A. Binhazim, A.A., Archibong, A.E., Chen, X., Ballard, B.R., Sairam, M.R., and Al-Hendy, A. (2012). Bone marrow transplantation restores follicular maturation and steroid hormones production in a mouse model for primary ovarian failure. PLoS One 7, e32462.

Guo, J.Q., Gao, X., Lin, Z.J., Wu, W.Z., Huang, L.H., Dong, H.Y., Chen, J., Lu, J., Fu, Y.F., Wang, J., et al. (2013). BMSCs reduce rat granulosa cell apoptosis induced by cisplatin and perimenopause. BMC Cell Biol. 14, 1-9.

Iorio, R., Castellucci, A., Ventriglia, G., Teoli, F., Cellini, V., Macchiarelli, G., and Cecconi, S. (2014). Ovarian toxicity: from environmental exposure to chemotherapy. Curr. Pharm. Des. 20, 5388-5397.

Johnson, J., Bagley, J., Skaznik-Wikiel, M., Lee, H.-J., Adams, G.B., Niikura, Y., Tschudy, K.S., Tilly, J.C., Cortes, M.L., Forkert, R., et al. (2005). Oocyte generation in adult mammalian ovaries by putative germ cells in bone marrow and peripheral blood. Cell 122, 303-315.

Kilic, S., Pinarli, F., Ozogul, C., Tasdemir, N., Naz Sarac, G., and Delibasi, T. (2013). Protection from cyclophosphamide-induced ovarian damage with bone marrow-derived mesenchymal stem cells during puberty. Gynecol. Endocrinol. 30, 135-140.

Kupcova Skalnikova, H. (2013). Proteomic techniques for characterisation of mesenchymal stem cell secretome. Biochimie 95, 2196-2211.

Lai, D., Wang, F., Chen, Y., Wang, L., Wang, Y., and Cheng, W. (2013). Human amniotic fluid stem cells have a potential to recover ovarian function in mice with chemotherapy-induced sterility. BMC Dev. Biol. 13, 34.

Lana, M.B.P., Straminsky, V., Onetto, C., Amuchastegui, J.M. Blanco, G., Galluzzo, L., Provenzano, S., and Nolting, M. (2010). What is really responsible for bone loss in spontaneous premature ovarian failure? A new enigma. Gynecol. Endocrinol. $26,755-759$.

Lee, O., Coathup, M., Goodship, A., and Blunn, G. (2005). Use of mesenchymal stem cells to facilitate bone regeneration in normal and chemotherapy-treated rats. Tissue Eng. 11, 17271735.

Lee, H.-J., Selesniemi, K., Niikura, Y., Niikura, T., Klein, R., Dombkowski, D.M., and Tilly, J.L. (2007). Bone marrow transplantation generates immature oocytes and rescues long-term fertility in a preclinical mouse model of chemotherapy-induced premature ovarian failure. J. Clin. Oncol. 25, 3198-3204.

Liu, T., Huang, Y., Guo, L., Cheng, W., and Zou, G. (2012). CD44+/ CD105+ human amniotic fluid mesenchymal stem cells survive and proliferate in the ovary long-term in a mouse model of chemotherapy-induced premature ovarian failure. Int. J. Med Sci. 9, 592-602.

Lue, Y., Erkkila, K., Liu, P.Y., Ma, K., Wang, C., Hikim, A.S., and Swerdloff, R.S. (2007). Fate of bone marrow stem cells transplanted into the testis: potential implication for men with testicular failure. Am. J. Pathol. 170, 899-908.

Mörse, H., Elfving, M., Lindgren, A., Wölner-Hanssen, P., Andersen, C.Y., and Øra, I. (2013). Acute onset of ovarian dysfunction in young females after start of cancer treatment. Pediatr. Blood Cancer 60, 676-681.

Maclaran, K., and Panay, N. (2011). Premature ovarian failure. J. Fam. Plann. Reprod. Health Care 37, 35-42.

Maruo, T., Laoag-Fernandez, J., Takekida, S., Peng, X., Deguchi, J., Samoto, T., Kondo, H., and Matsuo, H. (1999). Regulation of granulosa cell proliferation and apoptosis during follicular development. Gynecol. Endocrinol. 13, 410-419.

Matsuda, F., Inoue, N., Manabe, N., and Ohkura, S. (2011). Follicular growth and atresia in mammalian ovaries: regulation by survival and death of granulosa cells. J. Reprod. Dev. 58, 44-50.

Miyamoto, Y., Shimada, K., Sakaguchi, Y., and Miyamoto, M. (2007). Cisplatin (CDDP)-induced acute toxicity in an experimental model of hepatic fibrosis. J. Toxicol. Sci. 32, 311-319.

Muscari, C., Bonafè, F., Martin-Suarez, S., Valgimigli, S., Valente, S., Fiumana, E., Fiorelli, F., Rubini, G., Guarnieri, C., Caldarera, C.M., et al. (2013). Restored perfusion and reduced inflammation in the infarcted heart after grafting stem cells with a hyaluronan-based scaffold. J. Cell. Mol. Med. 17, 518-530.

Nayernia, K., Lee, J.H., Drusenheimer, N., Nolte, J., Wulf, G., Dressel, R., Gromoll, J., and Engel, W. (2006). Derivation of male germ cells from bone marrow stem cells. Lab. Invest. 86, 654-663. 
Nozaki, Y., Furubo, E., Matsuno, T., Fukui, R., Kizawa, K., Kozaki, T., and Sanzen, T. (2009). Collaborative work on evaluation of ovarian toxicity 6). Two-or four-week repeated-dose studies and fertility study of cisplatin in female rats. J. Toxicol. Sci. 34, SP7381.

Ohishi, M., and Schipani, E. (2010). Bone marrow mesenchymal stem cells. J. Cell. Biochem. 109, 277-282.

Rüster, B., Grace, B., Seitz, O., Seifried, E., and Henschler, R. (2005). Induction and detection of human mesenchymal stem cell migration in the 48-well reusable transwell assay. Stem Cells Dev. 14, 231-235.

Santos, N., Catao, C., Martins, N., Curti, C., Bianchi, M., and Santos, A. (2007). Cisplatin-induced nephrotoxicity is associated with oxidative stress, redox state unbalance, impairment of energetic metabolism and apoptosis in rat kidney mitochondria. Arch. Toxicol. 81, 495-504.

Schmidt, A., Ladage, D., Schinköthe, T., Klausmann, U., Ulrichs, C., Klinz, F.J., Brixius, K., Arnhold, S., Desai, B., and Mehlhorn, U. (2006). Basic fibroblast growth factor controls migration in human mesenchymal stem cells. Stem Cells $24,1750-1758$.

Selesniemi, K., Lee, H.-J., Niikura, T., and Tilly, J.L. (2009). Young adult donor bone marrow infusions into female mice postpone age-related reproductive failure and improve offspring survival. Aging 1, 49-57.
Siddik, Z.H. (2003). Cisplatin: mode of cytotoxic action and molecular basis of resistance. Oncogene 22, 7265-7279.

Sohni, A., and Verfaillie, C.M. (2013). Mesenchymal stem cells migration homing and tracking. Stem Cells Int. 2013, 130763.

Takehara, Y., Yabuuchi, A., Ezoe, K., Kuroda, T., Yamadera, R., Sano, C., Murata, N., Aida, T., Nakama, K., Aono, F., et al. (2013). The restorative effects of adipose-derived mesenchymal stem cells on damaged ovarian function. Lab. Invest. 93, 181-193.

Tannock, I.F., Ahles, T.A., Ganz, P.A., and van Dam, F.S. (2004). Cognitive impairment associated with chemotherapy for cancer: report of a workshop. J. Clin. Oncol. 22, 2233-2239.

Wang, S., Yu, L., Sun, M., Mu, S., Wang, C., Wang, D., and Yao, Y. (2013). The therapeutic potential of umbilical cord mesenchyma stem cells in mice premature ovarian failure. Biomed Res. Int. 2013, 690491.

Yuan, L., Wu, M.J., Sun, H.Y., Xiong, J., Zhang, Y., Liu, C.Y., Fu, L.L Liu, D.M., Liu, H.-Q., and Mei, C.-L. (2011). VEGF-modified human embryonic mesenchymal stem cell implantation enhances protection against cisplatin-induced acute kidney injury. Am. J. Physiol. Renal. Physiol. 300, F207-F218.

Zhang, A., Wang, Y., Ye, Z., Xie, H., Zhou, L., and Zheng, S. (2010). Mechanism of TNF- $\alpha$-nduced migration and hepatocyte growth factor production in human mesenchymal stem cells. J. Cell. Biochem. 111, 469-475. 